\begin{tabular}{lc}
\hline RELIABILITY \\
ENGINEERING \\
Reliability Engineering and System Safety $76(2002) 117-127$ & \& \\
SYSTEM \\
SAFETY \\
\hline \hline ww.elsevier.com/locate/ress
\end{tabular}

\title{
A maintenance policy for a system with multi-state components: an approximate solution
}

\author{
Ülkü Gürler ${ }^{a, *}$, Alev Kaya ${ }^{\mathrm{b}}$ \\ ${ }^{a}$ Department of Industrial Engineering, Bilkent University, Bilkent 06533, Ankara, Turkey \\ ${ }^{\mathrm{b}}$ Confida Consulting and Ventures, Ft Lauderdale, FL, USA
}

Received 27 April 2000; accepted 19 September 2001

\begin{abstract}
For maintenance and quality assessment purposes, various performance levels for both systems and components are identified, usually as a function of the deterioration. In this study, we consider a multicomponent system where the lifetime of each component is described by several stages, $(0, \ldots, S)$, which are further classified as good, doubtful, preventive maintenance due (PM due) and down. A control policy is suggested where the system is replaced when a component enters a PM due or a down state and the number of components in the doubtful states $(K, \ldots, S-2)$ is at least $N$. All maintenance activities are assumed to take negligible time. The exact description of the underlying stochastic model under the policy is very complicated. We therefore propose some approximations, which allow an explicit expression for the long run average cost function, which is minimized w.r.t. $(K, N)$ by numerical methods. Sensitivity of the model to system parameters and the performance of the approximation are investigated through several examples. (C) 2002 Elsevier Science Ltd. All rights reserved.
\end{abstract}

Keywords: Maintenance; Multi-state components; Markov processes; Randomization method

\section{Introduction}

In many real life situations, systems and their components are capable of assuming a whole range of performance levels, varying from perfect functioning to complete failure. There is a growing interest in the maintenance and replacement of multi-state systems indicated by the vast amount of existing literature. However, most of these studies consider single or two component systems. Multi-state, multicomponent maintenance systems have received more attention since 1990s, partly because of their applicability in the design and operation of computers and other service facilities as well as in the traditional areas like road maintenance, aircraft industry and oil production.

In maintenance optimization models the goal is to find the right compromise between preventive maintenance which extends the period of proper operation of the systems and corrective maintenance or replacement which replaces an old system by a new one. The decision of when to replace the system becomes rather involved if the system is composed of many components. In these situations, an

\footnotetext{
* Corresponding author. Tel.: +90-312-2901520; fax: +90-3122664054.

E-mail address: ulku@bilkent.edu.tr (U. Gürler).
}

important issue is to decide when to take a combined maintenance/replacement action on several components.

Although the special case of two components has been studied extensively, the literature for multicomponent systems is fairly recent. For two component systems, see Refs. [9,18,19,21,22] among others. For systems with arbitrary number of components, Özekici [15] provides a characterization of the structure of the optimal policy when component lifetimes have a certain dependency structure. Hsu [6] introduces a model for the preventive maintenance of a stochastically deteriorating serial production system and provides managerial implications for maintaining such systems. The results show that the operating characteristics of the production stations are interrelated and it is important to examine the joint effects of a maintenance policy on various stations of the production system simultaneously rather than studying each station separately. In another study, Jansen and Van der Duyn Schouten [7] analyze the optimal preventive maintenance for a production system consisting of independent and identical parallel production units with increasing failure rates. Berk and Moinzadeh [4] study a multi machine problem with limited repair capacity in which maintenance policies are based on both the number and the age of the operational machines.

The maintenance policies mentioned above utilize the age information for each component. See, e.g. Assaf and 
Shantikumar [2] and Ritchken and Wilson [17] who studied coordinated group maintenance policies based mainly on the number of failed components in the system. For some recent studies on multistate systems see also Refs. [11,12,16].

In our study, we consider a multicomponent series system where the independent and identical components are allowed to go through several stages during their life spans corresponding to different depreciation levels. Series models with identical components where the components are subject to deterioration can be encountered in relays or networks, where the failure of a single component results in system failure. We provide below some examples.

Railroad tracks. This example is provided by Van der Duyn Schouten and Vanneste [20] who considered a system where four possible states are identified for each component: good, doubtful, preventive maintenance due, and failed. Their model was inspired by the maintenance of a regional railroad track. Depending on the waives occurring in the rails, a certain segment of a section is classified in one of the three possible states: bad, doubtful, and good. Due to safety regulations, a bad segment of a section has to be maintained without delay and requires specific equipment, which has a high cost. Once this equipment is hired for a certain time, it is feasible to repair other segments beyond a deterioration level.

Telecommunication systems. Relays or repeaters in telecommunication systems operating with radio or microwaves can be considered as a series system with identical components. In these examples, the dish antennas or optical/ electrical amplifiers located at usually equally distant points in a region can be modeled as identical components although they might have slight differences in their brand or model. In these systems system failure can be immediately detected and the stages of the components may be continuously detected by the quality of the transferred signal.

Infrastructure systems. Segments of asphalted highways where stages can be identified due to cracking or textural damage; or drain systems where the damage and amount of leakage at a given segment can be used to define the component stages are also examples of series systems with identical components. See also Noorwijk and Klatter [13] who studied the optimal maintenance of the block mats of a barrier on individual basis. When the entire barrier is considered the block mats can be taken as the identical components of a series system.

Our study considers an extension of the model given by Van der Duyn Schouten and Vanneste [20], by allowing the lifetime of each component to assume several stages. The main motivation behind such an extension is that with the increasing functional and mechanical complexity of the devices of high technology, the state descriptions corresponding to the degradation level also becomes complicated. Parallel to this, advanced measurement devices provide us with more detailed information about the performance of systems and components. By introducing $S$ different stages, our model aims to grasp this available information and utilize it in an effective way. For relatively simple products, where the state of a component can be characterized by one or two attributes, restricting the number of possible states may bring substantial simplifications in the analysis of the model and therefore would be advisable. However for more complex systems, several performance measures can be used to describe the status of the system, which can be transferred to a single ordered index, which indicates the current state of the system. The model suggested in this paper allows to have as many states as required by the nature of the system. As a second possible generalization worthwhile to mention is that our model can accommodate Erlang or phase-type sojourn times in each state (see Refs. [1,3]). Although the ideas in this manuscript are illustrated in terms of exponential sojourn times for notational simplicity, the extension to such distributions is straightforward by augmenting the state space. Such an extension provides an important flexibility, since besides being a rich class for modeling lifetime distributions, they provide approximations to other distribution. Specifics of such an extension is discussed in Section 4.

We propose a maintenance policy which is based on the number of components in 'doubtful' states. A renewal theoretical approach is adopted to derive the long run expected cost function per unit time, which is aimed to be optimized w.r.t. the decision variables. The objective function is derived as a function of the limiting state probabilities of the corresponding stochastic process under the proposed maintenance policy. The analytical derivations of these steady state probabilities are very complicated and therefore we propose an approximation. Validation of the approximation is done by comparing the results with those obtained from simulations. The results of the cases examined indicate that the suggested maintenance policy achieves significant reductions on the maintenance costs, if instead of a group maintenance policy; the components are replaced whenever they fail.

The organization of the paper is as follows. In Section 2, the model description and the preliminaries are presented. In Section 3, results concerning the long run behavior of the system are derived. In Section 4, implementation of the results are illustrated and the comparisons of the approximated and simulated models are provided. Finally in Section 5, concluding remarks are given together with further research directions.

\section{Model description and preliminaries}

We now formally describe the model discussed in Section 1. Consider a system composed of $M$ identical and independently operating components which are connected in series. The condition of each component is characterized by $S+1$ possible states, where 0 is the best state and $S$ corresponds to the down state. For maintenance purposes these states are 


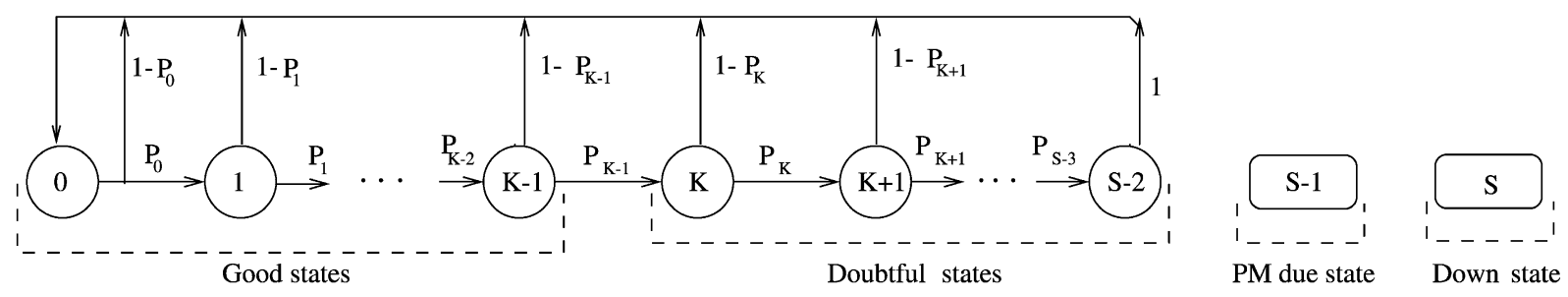

Fig. 1. Behavior of a component.

further classified into four categories in the following way: states 0 to $K-1$ are good, states $K$ to $S-2$ are doubtful, state $S-1$ is preventive maintenance due (PM due), and state $S$ is down. A component in a good or a doubtful state can either grow one state older or break down with a known probability. That is, from state $l, l=1, \ldots, S-2$, a transition occurs to either state $l+1$ or to state $S$ (down), with probabilities $p_{l}$ and $1-p_{l}$, respectively (see Fig. 1). Upon entrance to a PM due state, an immediate preventive maintenance is carried out and, upon entrance to the down state an immediate corrective maintenance is done.

The sojourn time at state $l$ is distributed as exponential with parameter $v_{l}$ and according to the instantaneous repair assumption, the sojourn in PM due and down states are zero. Therefore in practice only the states from zero to $S-2$ are operational. As to the cost parameters, we assume that corrective replacement is more costly than preventive replacement and system replacement cost is less than replacing each component preventively or correctively. The cost of preventive and corrective component replacements are $c_{1}$ and $c_{2}$, respectively, and the system replacement cost is $c_{3}$. The assumptions above then imply that $c_{2}>c_{1}$ and $c_{3}<M c_{1}$. We propose the following control policy for the system described above.

Control policy. The system is replaced when a component enters a PM due or the down state and the number of components in doubtful states $K, \ldots, S-2$ at that moment is at least $N$.

This policy is based on two decision variables, $K$ and $N$ where the former one dictates the effective classification of the states and the second one is the threshold value for the number of doubtful components beyond which it is not economical to further wait for the system replacement. Our aim is to find the optimal values of $K$ and $N$ which minimizes the long run average cost function per unit time.

\section{Remarks.}

1. In our model both the PM and corrective maintenance bring the component back into state 0 and are assumed to be instantaneous so that the system operation is not interrupted. In practice this assumption requires that the maintenance times can be considered negligible in comparison to the component or system lifetimes. Alternatively, if the maintenance times are not negligible, the proposed model is applicable to the optimization of the operating time cost, which is considered as an alternative measure of reliability as discussed by Kumar et al. [10].

2. Our control policy requires that the exact state of each component is immediately known. This assumption holds for systems, which can be controlled/observed continuously, such as the telecommunication or power distribution systems where the states may be defined with respect to the quality of the output, which can be continuously observed by remote control devices. In reality, inspections can be made on a periodic basis and the proposed model may provide a good approximation if the inspection intervals are small relative to the component lifetime.

3. The system introduced above is described as a series system. However, with the assumption of negligible maintenance time, the model also applies to parallel systems with identical components. Hence the proposed model is also applicable to production environments with identical parallel machines, computer networks, telephone networks etc., for which the state classifications can be made more easily according to the amount of output or usage units. Similarly, in such systems current status data can be immediately available.

4. As an alternative formulation, suppose the states are classified differently as: $[0, K-1]$ : Good, $[K, S-$ $J]$ : Doubtful, $[S-J+1, S-1]$ PM Due and $S$ : Down. Since the preventive maintenance is done when a component enters the PM due state and the maintenance brings the component to the good as new state immediately, the states $S-J+2, S-J+$ $3, \ldots, S-1$ will never be visited, which amounts to reducing the effective number of states from $S$ to $S-J$ instead of $S-2$ in the current model. This case can easily be handled by the present model and as an extension of our model, the value of $J$ can also be optimized.

\subsection{Stochastic analysis}

The exact stochastic nature of the system operated under the proposed control policy can be described by a multi dimensional Markov process $(\mathrm{MP}) X(t)=$ $\left\{X_{0}(t), X_{1}(t), \ldots, X_{S-2}(t)\right\}$, where $X_{i}(t)$ is the number of 
components that are in their $i$ th state at time $t$. Then the replacement time can be described in terms of the first passage time of this MP to a particular state. Although theoretically it should be possible to carry out such an analysis by using advanced techniques for multidimensional MP, it is easily seen that this analysis would be complicated, especially when the number of components and the number of states are large, inducing a very high dimensional state space. We therefore employ an alternative approach albeit at a cost of an approximate solution. The aim of our approach is to provide an approximation, which reduces the dimension of the problem. The proposed method involves two main approximations as described below. First let $W(t)$ denote the number of components in the doubtful states at time $t, t \geq 0$. Then, $\{W(t), t \geq 0\}$ is a process defined by a subset $\left(X_{K}\right.$ to $X_{S-2}$ ) of components of $X(t)$ and as such, it is no longer a MP. The Markov property disappears since the transition rates of $W(t)$ depends on the number of components at other states at time $t$, which is a multidimensional random variable. If these transition rates were known, then $\{W(t), t \geq 0\}$ would behave as a MP defined on $\{0, \ldots, M ; \delta\}$, where $\delta$ corresponds to the system replacement. A transition diagram for $\{W(t), t \geq 0\}$ is given in Fig. 2.

Here, $\lambda_{i}, 0 \leq i \leq M-1 ; i \neq N$, is the rate of transitions due to which the number of doubtful components increases from $i$ to $i+1, \mu_{i}, 0 \leq i \leq N$, is the rate of transitions due to which the number of doubtful components decreases from $i$ to $i-1$, and $\alpha_{i}$ the rate of transitions due to which the number of doubtful components remains constant at $i$. Also, for $N+1 \leq i \leq M, \beta_{i \delta}$ is the rate of transitions due to which a system replacement is triggered given that there are $i$ doubtful components. We define $\lambda_{N_{1}}$ as the rate of transitions due to which the number of doubtful components increase from $N$ to $N+1$. This transition is different in nature than the previous ones in that once it is realized; the process does not come back to earlier states. The total rate with which the process leaves state $N$ is defined as $\lambda_{N}=$ $\lambda_{N_{1}}+\beta_{N, \delta}$. Note that, as long as the number of doubtful components does not reach $N+1$ and a system replacement does not take place, the behavior of $\{W(t), t \geq 0\}$ defined on
$\{0, \ldots, N+1, \delta\}$ is analogous to a birth and death process with absorbing states $N+1$ and $\delta$. From the moment at which the number of doubtful components is $N+1$ until the system replacement, it behaves similar to a Markov chain defined on the states $\{N+1, \ldots, M, \delta\}$, where $\delta$ is the absorbing state and the process makes a transition either to the next state or to the absorbing one.

Our approach consists of a two stage approximation. The first stage is to treat $\{W(t), t \geq 0\}$ as a MP as described above, and the second is to get approximate expressions for the transition rates of this process, the details of which are given in Section 3. The main idea in the second stage is to approximate the rates by appropriate functions of conditional expectations of the exact MP $X(t)$.

Once the expressions for the transition rates are obtained, results on birth and death processes with absorbing states can be utilized to yield the expressions for the long run operating characteristics of the system. To this end, it is also useful to make the following definitions: a backward jump of $\{W(t), t \geq 0\}$ is a transition from node $i$ to $i-1$, a dummy jump of $\{W(t), t \geq 0\}$ is a transition from node $i$ to itself. Therefore, a backward jump corresponds to transition of a single component from the doubtful state via an instantaneous bad or down state to a good state, whereas a dummy jump correspond to the transition of a single component from a good state via an instantaneous down state back to a good state. Note also that, backward jumps are associated with either a preventive or a corrective replacement whereas dummy jumps are always associated with a corrective replacement.

Throughout the study, we assume that all components are at state 0 at time $t=0$, implying $W(0)=0$. Now, define $\tau$ as the expected time until a system replacement takes place or the number of doubtful components reaches $N+1$, whichever occurs first; $\kappa$ and $\varphi$ as the expected number of backward and dummy jumps of $\{W(t), t \geq 0\}$ before a system replacement takes place and $\sigma$ as the expected time until a system replacement takes place, given that there are $N+1$ doubtful components at $t=0$. Then Proposition 1 below can be obtained by referring to Karlin and Taylor [8] (see also Ref. [20]). For $2 \leq i \leq N$, let $\rho_{0}=\lambda_{0}^{-1}$,

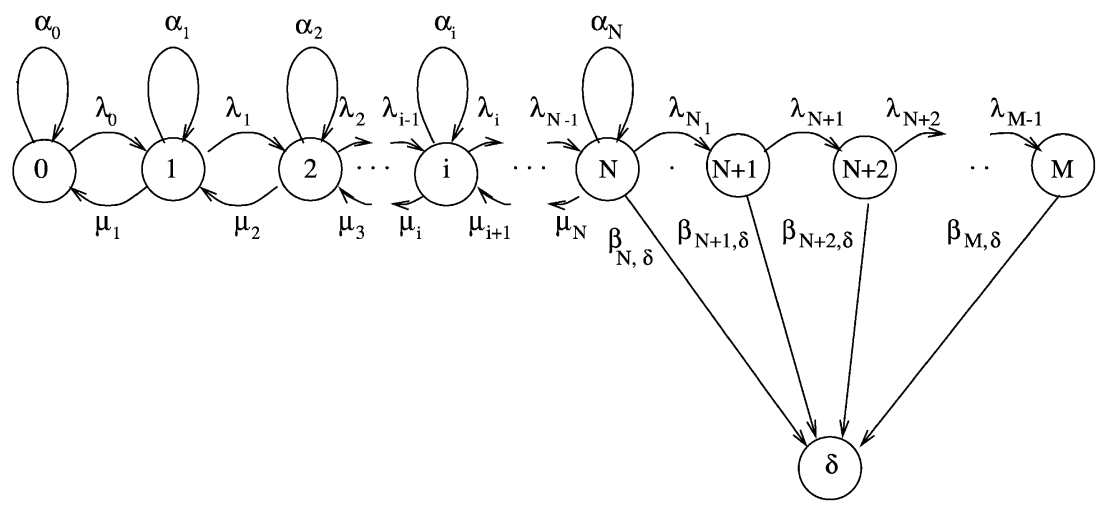

Fig. 2. Transition rate diagram of $\{W(t), t \geq 0\}$. 
$\rho_{1}=\mu_{1}^{-1}$ and

$\rho_{i}=\frac{\lambda_{1} \lambda_{2} \cdots \lambda_{i-1}}{\mu_{1} \mu_{2} \cdots \mu_{i}}$

with $\rho_{0}=\lambda_{0}^{-1}$ and $\rho_{1}=\mu_{1}^{-1}$.

\section{Proposition 1.}

$\varphi=\sum_{j=0}^{N} \frac{1}{\lambda_{j} \rho_{j}} \sum_{l=0}^{j} \alpha_{l} \rho_{l}$

$\kappa=\sum_{j=0}^{N} \frac{1}{\lambda_{j} \rho_{j}} \sum_{l=0}^{j} \mu_{l} \rho_{l}$

$\tau=\sum_{j=0}^{N} \frac{1}{\lambda_{j} \rho_{j}} \sum_{l=0}^{j} \rho_{l}$

$\sigma=\sum_{j=N+1}^{M} \frac{1}{\lambda_{j}+\beta_{j \delta}}\left[\prod_{l=N+1}^{j-1} \frac{\lambda_{l}}{\lambda_{l}+\beta_{l \delta}}\right]$

\section{Average cost function and proposed approximations}

\subsection{The cost function}

In this section, an expression for the long run average cost per unit time is derived for the model under study. Consider an $M$ dimensional stochastic process described by the states of the $M$ components at time $t$. Then, under the proposed policy, the system replacement times constitute regeneration points for this process and we define a regenerative cycle as the time that elapses between two successive system replacements. Using the renewal reward theorem, the long run average cost per unit time can be expressed as the ratio of expected cost incurred in a cycle to expected length of a cycle. Theorem 1 below provides an approximate long run cost function, which is aimed to be minimized w.r.t. the decision variables $K$ and $N$.

The expression for the long run average cost per unit time is derived as a function of the steady state probabilities of the stochastic process representing the state of a component under the proposed control policy. Although the behaviors of the components are not stochastically independent, they are identical. Let then $\pi_{l}, l=0, \ldots, S-2$, denote the limiting probability that a component is at state $l$ under the given control policy. The exact values of these probabilities would be obtained from the long run behavior of a multidimensional MP representing the state of each component at any time. However as mentioned before, we aim to reduce the dimension of the problem. Therefore an approximation algorithm to evaluate the $\pi_{l}$ 's will be provided below. Let

$\pi_{\mathrm{D}}=\sum_{l=K}^{S-2} \pi_{l}, \quad \pi_{\mathrm{G}}=\sum_{l=0}^{K-1} \pi_{l}$,

$\omega(n)=\sum_{l=0}^{n} \pi_{l} v_{l}\left(1-p_{l}\right), \quad n=0, \ldots, K-1$,

$\bar{\omega}(m)=\sum_{l=K}^{m} \pi_{l} v_{l}\left(1-p_{l}\right), \quad m=K, \ldots, S-2$

and

$\tilde{p}_{K}=\frac{\pi_{S-2} p_{S-2, S-1}}{\pi_{K} p_{K, S}+\cdots+\pi_{S-3} p_{S-3, S}+\pi_{S-2}}$

In these expressions, $\pi_{\mathrm{D}}$ refers to the long run probability that a component is in one of the doubtful states $K, \ldots, S-$ $2 ; \omega(n)$ to the total failure rate of the states 0 to $n, n=$ $0, \ldots, K-1$; and $\bar{\omega}(m)$ to the total failure rates of the states $K$ to $m, m=K, \ldots, S-2$.

Let $\mathrm{AC}(K, N)$ be the long run average cost function obtained under the approximate model described above. Referring to the expressions (1)-(4) of Section 2.1, we have:

\section{Theorem 1.}

$\mathrm{AC}(K, N)=\frac{c_{2}\left[\varphi+\left(1-\tilde{p}_{K}\right) \kappa\right]+c_{1} \tilde{p}_{K} \kappa+c_{3}}{\tau+P\left(T_{1} \neq 0\right) \sigma}$

where

$P\left(T_{1} \neq 0\right)=\frac{\pi_{K-1} v_{K-1} p_{K-1}}{\omega(K-2)+\pi_{K-1} v_{K-1}}$

Proof. Let $T$ denote the length of a cycle, which we can write as $T=T_{0}+T_{1}$, where $T_{0}$ is the entrance time of $\{W(t), t \geq 0\}$ into $\delta$ or node $N+1$ (which ever occurs first), and $T_{1}$ is the time between $T_{0}$ and the entrance of $\{W(t), t \geq 0\}$ into $\delta$ in Fig. 2. That is, $T_{0}$ represents the moment at which $\{W(t), t \geq 0\}$ leaves the set $\{0, \ldots, N\}$ via entrance into node $N+1$ or $\delta$, while $T_{1}$ denotes the time-interval between $T_{0}$ and a system replacement. Then

$E T=\tau+P\left(T_{1} \neq 0\right) \sigma$

Note that $P\left(T_{1} \neq 0\right)$ can be considered as the probability of termination of the system when there are more than $N$ doubtful components and it can be represented as the ratio of the rate of transitions from node $N$ to $N+1$, to transition rate $\lambda_{N}$. We know that $\lambda_{N}$ is the sum of the rates of transitions from node $N$ to $N+1$ and from node $N$ to system 
replacement. Therefore

$P\left(T_{1} \neq 0\right)$

$$
=\frac{\frac{(M-N)}{\pi_{\mathrm{G}}} \pi_{K-1} v_{K-1} p_{K-1}}{\frac{(M-N)}{\pi_{\mathrm{G}}} \pi_{K-1} v_{K-1} p_{K-1}+\frac{(M-N)}{\pi_{\mathrm{G}}} \omega(K-1)}
$$

from which Eq. (6) follows.

On $[0, T]$, costs are incurred only on $\left[0, T_{0}\right]$ (costs of corrective and preventive component replacements) and at time $T$ (system replacement costs). Every dummy jump of $\{W(t), t \geq 0\}$ corresponds to a corrective replacement and every backward jump of $\{W(t), t \geq 0\}$ corresponds to a corrective component replacement with probability $1-$ $\tilde{p}_{K}$, and to a preventive component replacement with probability $\tilde{p}_{K}$. Therefore, the expected total cost in a cycle is

$E C(T)=c_{2}\left[\varphi+\left(1-\tilde{p}_{K}\right) \kappa\right]+c_{1} \tilde{p}_{K} \kappa+c_{3}$

which completes the proof.

Optimization of the objective function $\mathrm{AC}(K, N)$ is done via numerical methods which is presented in Section 3.2.

\subsection{Approximations}

Approximations for the rates of $W(t)$. Let $n_{l}$ and $n_{\mathrm{D}}$ refer to the number of components in state $l$ and in doubtful states respectively and $E[\cdot \mid \cdot]$ stand for conditional expectation. For the evaluation of the average cost function, we propose to approximate the rates of the process $\{W(t), t \geq 0\}$ as follows:

$\lambda_{i} \sim E\left[n_{K-1} \mid n_{\mathrm{D}}=i\right] v_{K-1} p_{K-1}$

$\lambda_{N_{1}} \sim E\left[n_{K-1} \mid n_{\mathrm{D}}=N\right] v_{K-1} p_{K-1}$

$\lambda_{N} \sim \sum_{l=0}^{K-2} E\left[n_{l} \mid n_{\mathrm{D}}=N\right] v_{l}\left(1-p_{l}\right)+E\left[n_{K-1} \mid n_{\mathrm{D}}=N\right] v_{K-1}$

$\alpha_{i} \sim \sum_{l=0}^{K-1} E\left[n_{l} \mid n_{\mathrm{D}}=i\right] v_{l}\left(1-p_{l}\right)$

$\mu_{i} \sim \sum_{l=K}^{S-3} E\left[n_{l} \mid n_{\mathrm{D}}=i\right] v_{l}\left(1-p_{l}\right)+E\left(n_{S-2} \mid n_{\mathrm{D}}=i\right] v_{S-2}$

$\beta_{i \delta} \sim \sum_{l=0}^{S-3} E\left[n_{l} \mid n_{\mathrm{D}}=i\right] v_{l}\left(1-p_{l}\right)+E\left[n_{S-2} \mid n_{\mathrm{D}}=i\right] v_{S-2}$

$\beta_{N \delta} \sim \sum_{l=0}^{K-1} E\left[n_{l} \mid n_{\mathrm{D}}=N\right] v_{l}\left(1-p_{l}\right)$
Evaluating the above expressions, as partially proved in the appendix, we get the following rates:

Lemma 1.

(a)

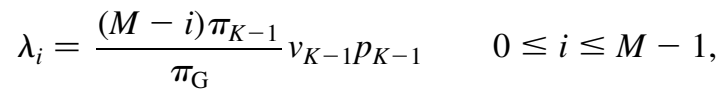

$i \neq N$

(b)

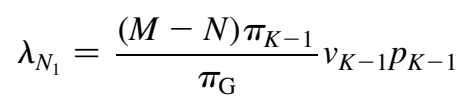

(c)

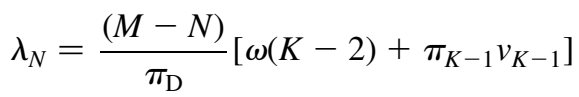

(d)

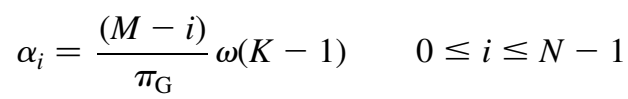

(e) $\quad \alpha_{N}=0$

$$
\mu_{i}=\frac{i}{\pi_{\mathrm{D}}}\left[\bar{\omega}(S-3)+\pi_{S-2} v_{S-2}\right] \quad 0 \leq i \leq N
$$

(g)

$\beta_{i \delta}=\frac{(M-i)}{\pi_{\mathrm{G}}} \omega(K-1)+\frac{i}{\pi_{\mathrm{D}}}\left[\bar{\omega}(S-3)+\pi_{S-2} v_{S-2}\right]$

$N+1 \leq i \leq M$

(h)

$$
\beta_{N \delta}=\frac{(M-N)}{\pi_{\mathrm{G}}} \omega(K-1)
$$

The cost function is evaluated by using the rates given above in conjunction with the expressions given in Proposition 1.

\subsubsection{Approximations for the limiting probabilities}

In the system under consideration, although the components are operating independently of each other, due to the total replacement policy, some dependency is imposed. However their steady state probabilities will still be identically distributed. As described earlier, let $\pi_{j}, j=$ $1,2, \ldots, S-1$, stand for the limiting probability that a component is in state $j$, under the control policy. We introduce below an iterative algorithm, which approximates these steady state probabilities. This algorithm is based on the transient probabilities of a process corresponding to the state of a single component, if a control policy is not imposed on the system. In particular, suppose we have a series system of $M$ components which have identical lifetimes and operate independently of each other. As before a component can go through several stages before it fails and it is repaired instantaneously upon failure. No further system replacement policy is adopted. Then the behavior of components can be expressed as independent and identical 
processes and it is sufficient to consider a single component. Let $\{X(t), t \geq 0\}$ be the process representing the state of this component at time $t$ with the transient probability function given by $\gamma_{l}(t)$ and the limiting probabilities, $\gamma_{l}=$ $\lim _{t \rightarrow \infty} \gamma_{l}(t), l=0, \ldots, S-2$. When the suggested control policy is imposed on the system, the process $\{X(t), t \geq 0\}$ is interrupted at the time of system replacements and therefore steady states for the components may not be achieved within a cycle and need to be modified. Based on the usual interpretation of the steady state probabilities as the proportion of time that the system spends in that state and recalling that the times between system replacement constitute regenerative cycles, we propose the following iterative approximation for the $\pi_{j}$ s, where $\hat{E}(T)$ corresponds to an estimate of the expected cycle length

$\hat{\pi}_{l} \frac{1}{\hat{E}[T]} \int_{0}^{\hat{E}[T]} \gamma_{l}(t) \mathrm{d} t$

This approximation incorporates the impact of the control policy on $\hat{\pi}_{l} s$ through the estimated values $\hat{E}[T]$. The implementation requires the estimation of the transient probabilities $\gamma_{l}(t)$, and the expected cycle length $E[T]$, which in turn is expressed as a function of the limiting $\pi_{l} \mathrm{~s}$ and hence an iterative procedure is needed. We start with an initial value of $\hat{E}[T]$ and evaluate (14) to obtain $\hat{\pi}_{l}$, which are then used to get an updated value for $\hat{E}[T]$, via the expression given in the denominator of Eq. (5). The process is repeated until convergence occurs. For the initial value of $\hat{E}[T]$ we use the expected sojourn time in the best state since the cycle length is at least as long as that time period. However, our experience with other choices of starting values indicate that the optimal value is insensitive to these initial values.

\section{Remarks.}

1. This algorithm can be improved by re integrating the above integral w.r.t. the cycle length (instead of its expectation) which however requires the estimation of the distribution of the cycle length. One possibility could be to use an exponential distribution with rate $\lambda=$ $1 / \hat{E}[T]$. However as discussed in Section 4, the algorithm proposed above performs quite well.

2. If $M$ and $N$ are large, the time until system replacement may get long enough so that the $\{X(t), t \geq 0\}$ tends to its steady state and $\gamma_{l}$ may provide a good approximation for $\pi_{l}$. Correspondingly, $E[T]$, the expected time until system replacement will also tend to attain large values for large $M$ and $N$.

For the evaluation of the transient probabilities, $\gamma_{l}(t)$, a randomization method is used. This method is implicitly indicated within the algorithm presented below, further details of it can be found in Ref. [5]. The notation for the vectors used in the algorithm is as follows:

$\hat{\pi}=\left[\hat{\pi}_{0}, \hat{\pi}_{1}, \ldots, \hat{\pi}_{S-2}\right]^{\mathrm{T}}$,

$\gamma(t)=\left[\gamma_{0}(t), \gamma_{1}(t), \ldots, \gamma_{S-2}(t)\right]^{\mathrm{T}}$

Let $C$ stand for the matrix of the infinitesimal generator for the Markov process $\{X(t), t \geq 0\}$, which is a sparse matrix with non-zero elements only in the first column, in the main diagonal and the secondary diagonal to the right of the main one, and rows sum zero. An example of $C$ is given below for a specific case with $S=5$.

$C=\left[\begin{array}{cccc}-v_{0} p_{0} & v_{0} p_{0} & 0 & 0 \\ v_{1}\left(1-p_{1}\right) & -v_{1} & v_{1} p_{1} & 0 \\ v_{2}\left(1-p_{2}\right) & 0 & -v_{2} & v_{2} p_{2} \\ v_{3} & 0 & 0 & -v_{3}\end{array}\right]$

Then the iterative algorithm proposed in the Section 3.2 can be written explicitly as

Algorithm

1. Provide an initial value for $\hat{E}[T]$

2. Assign $\hat{\pi}_{\text {old }}:=1$ and $\hat{\pi}:=0$

3. While $\left|\hat{\pi}_{\text {old }}-\hat{\pi}\right|>\epsilon$ do

3.1. $\hat{\pi}_{\text {old }}:=\hat{\pi}$

3.2. Determine $\hat{\pi}$ with current $\hat{E}[T]$

3.2.1. Evaluate $\gamma(t)$ using randomization method

3.2.1.1. Set $\alpha:=\max _{i}\left|c_{i i}\right|$

3.2.1.2. Set $P:=\frac{1}{\alpha} C+I$

3.2.1.3. Determine $p$ such that

$1-\sum_{k=0}^{p} \mathrm{e}^{-\alpha t \frac{(\alpha t)^{k}}{k !} \leq \epsilon}$

3.2.1.4. $\phi(0):=\gamma(0)$

3.2.1.5. For $n=0$ to $p-1$ do

$\phi(n+1):=\phi(n) P$

3.2.1.6. $\gamma(t):=\sum_{n=0}^{p} \phi(n) \mathrm{e}^{-\alpha t \frac{(\alpha t)^{n}}{n !}}$

3.3. Determine $\mathrm{AC}(K, N)$ and $\hat{E}[T]$ using Theorem 1 .

\section{Numerical results}

In this section, proposed procedures are implemented through several examples. Due to the large number of parameters involved in the model such as the rates of the sojourn times, transition probabilities of the states and the cost figures, it is hard to make an extensive sensitivity study for all parameters. Therefore, the numerical investigation has focused mainly on two issues. First one is the evaluation of the performance of proposed approximation method for the limiting state probabilities. This is done by comparing 
the resulting steady state distribution to that obtained from simulations. Secondly, it is aimed to analyze the sensitivity of the control policy with respect to some basic system parameters.

As mentioned earlier, the methods proposed in this paper easily extends to the case where the sojourn time in each state has an Erlang distribution. This amounts to an enlargement in the state space of the underlying processes. In particular, suppose that the sojourn time at state $l, l=$ $0,1, \ldots, S-2$, has an Erlang distribution with $k_{l}$ stages (i.e. $k_{l}$-Erlang) and the rate of transition from one stage to another given by $v_{l}$. Then $k_{l}$ denotes the number of exponential stages in state $l$ and the foregoing analysis holds with the number of effective states extended from $S-1$ to $\sum_{l=0}^{S-2} k_{l}$. To include the implementation of this extension, the sojourn times in the following examples are taken to be Erlang.

The size of the problem becomes very large as $M$ and $S$ increase, which in turn leads to very long simulation runs. Therefore the results of the exhaustive search for optimal $(K, N)$ pair is illustrated only for the first example, and the results of the second example are given for a subset of $(K, N)$ pairs. The second example is further elaborated by varying the number of components, the cost figures and the transition probabilities. In the tables and the figures displayed below, $\hat{\pi}$ and $\tilde{\pi}$ denote the vector of approximated and simulated long run probability distribution over $S-1$ stages. The quantities $\epsilon_{\min }, \epsilon_{\max }$, and $\epsilon_{\mathrm{av}}$ refer to the minimum, maximum, and the average absolute differences between the components of the vectors $\hat{\pi}$ and $\tilde{\pi}$. Also, for the ease of presentation, the value of $\operatorname{AC}(K, N)$ is denoted by $c$, where as above $\hat{c}$ and $\tilde{c}$ correspond to average costs calculated using approximated and simulated probabilities, respectively. Finally, the $\%$ error, is the percentage of the absolute difference between $\hat{c}$ and $\tilde{c}$ with respect to $\tilde{c}$.

Example 1. Consider a simple system with three $(M=3)$ components, where each component can be in one of the five states, i.e. $S=4$. The sojourn times in states 0,1 , and 2
Table 1

Example 1, approximated and simulated results

\begin{tabular}{|c|c|c|c|c|c|c|c|}
\hline \multicolumn{2}{|c|}{$K=1, N=1$} & \multicolumn{2}{|c|}{$K=1, N=2$} & \multicolumn{2}{|c|}{$K=2, N=1$} & \multicolumn{2}{|c|}{$K=2, N=2$} \\
\hline$\hat{\pi}$ & $\tilde{\pi}$ & $\hat{\pi}$ & $\tilde{\pi}$ & $\hat{\pi}$ & $\tilde{\pi}$ & $\hat{\pi}$ & $\tilde{\pi}$ \\
\hline 0.850 & 0.853 & 0.818 & 0.827 & 0.827 & 0.824 & 0.819 & 0.818 \\
\hline 0.108 & 0.118 & 0.127 & 0.129 & 0.122 & 0.132 & 0.132 & 0.132 \\
\hline 0.037 & 0.029 & 0.048 & 0.044 & 0.045 & 0.044 & 0.048 & 0.049 \\
\hline$\epsilon_{\min }$ & 0.003 & $\epsilon_{\min }$ & 0.002 & $\epsilon_{\min }$ & 0.001 & $\epsilon_{\min }$ & 0.001 \\
\hline$\epsilon_{\max }$ & 0.010 & $\epsilon_{\max }$ & 0.009 & $\epsilon_{\max }$ & 0.010 & $\epsilon_{\max }$ & 0.001 \\
\hline$\epsilon_{\mathrm{av}}$ & 0.007 & $\epsilon_{\mathrm{av}}$ & 0.005 & $\epsilon_{\mathrm{av}}$ & 0.005 & $\epsilon_{\mathrm{av}}$ & 0.001 \\
\hline$\hat{c}$ & $\tilde{c}$ & $\hat{c}$ & $\tilde{c}$ & $\hat{c}$ & $\tilde{c}$ & $\hat{c}$ & $\tilde{c}$ \\
\hline 0.351 & 0.356 & 0.427 & 0.424 & 0.415 & 0.411 & 0.467 & 0.462 \\
\hline \multicolumn{2}{|c|}{$\%$ error $=1.4$} & \multicolumn{2}{|c|}{$\%$ error $=0.71$} & \multicolumn{2}{|c|}{$\%$ error $=0.97$} & \multicolumn{2}{|c|}{$\%$ error $=1.1$} \\
\hline
\end{tabular}

are 3-stage Erlang with rates $v_{0}=0.1, v_{1}=0.5$, and $v_{2}=$ 0.8. Sojourn times in states 3 and 4 are instantaneous. Preventive, corrective maintenance, and system replacement costs are $c_{1}=1.5, c_{2}=2, c_{3}=2.5$, respectively. Transition probabilities among states are given as $p_{0}=$ $0.8, p_{1}=0.6, p_{2}=0.4$, which may reflect an increasing hazard rate since the probability of failure increases as the component passes through the states. In this example ( $S-$ 2) $\times(M-1)=4$, and the results for these cases are displayed in Table 1. We observe that the approximation algorithm works quite satisfactorily, yielding limiting probabilities very close to those obtained from the simulations. The maximum average difference between the approximate and the simulated probabilities appear to be 0.007 , which is well acceptable. The impact of the approximation on the values of the average cost function seems to yield higher differences between analytical and simulated results, which however still stays within acceptable levels.

Example 2. In this example, the number of components is $M=7$ and $S=6$. Sojourn times in states $0,1,2$ and 3 are exponential with rates, $v_{0}=v_{1}=v_{2}=0.1$, and $v_{3}=1$, respectively, and sojourn time in state 4 is 3-Erlang with rate $v_{4}=1$. Recall that sojourn times in states 5 and 6 are

Table 2

Example 2, approximated and simulated results

\begin{tabular}{|c|c|c|c|c|c|c|c|c|c|}
\hline \multicolumn{2}{|c|}{$K=1, N=3$} & \multicolumn{2}{|c|}{$K=1, N=4$} & \multicolumn{2}{|c|}{$K=1, N=5$} & \multicolumn{2}{|c|}{$K=2, N=2$} & \multicolumn{2}{|c|}{$N=2, N=3$} \\
\hline$\hat{\pi}$ & $\tilde{\pi}$ & $\hat{\pi}$ & $\tilde{\pi}$ & $\hat{\pi}$ & $\tilde{\pi}$ & $\hat{\pi}$ & $\tilde{\pi}$ & $\hat{\pi}$ & $\tilde{\pi}$ \\
\hline 0.547 & 0.553 & 0.507 & 0.515 & 0.445 & 0.476 & 0.481 & 0.493 & 0.412 & 0.421 \\
\hline 0.304 & 0.302 & 0.315 & 0.310 & 0.323 & 0.318 & 0.320 & 0.327 & 0.323 & 0.346 \\
\hline 0.132 & 0.133 & 0.158 & 0.159 & 0.205 & 0.189 & 0.177 & 0.163 & 0.235 & 0.212 \\
\hline 0.011 & 0.012 & 0.014 & 0.015 & 0.019 & 0.016 & 0.016 & 0.015 & 0.022 & 0.019 \\
\hline 0.000 & 0.001 & 0.000 & 0.002 & 0.003 & 0.002 & 0.002 & 0.001 & 0.003 & 0.000 \\
\hline$\epsilon_{\min }$ & 0.001 & $\epsilon_{\min }$ & 0.001 & $\epsilon_{\min }$ & 0.001 & $\epsilon_{\min }$ & 0.001 & $\epsilon_{\min }$ & 0.003 \\
\hline$\epsilon_{\max }$ & 0.006 & $\epsilon_{\max }$ & 0.008 & $\epsilon_{\max }$ & 0.031 & $\epsilon_{\max }$ & 0.014 & $\epsilon_{\max }$ & 0.023 \\
\hline$\epsilon_{\mathrm{av}}$ & 0.002 & $\epsilon_{\mathrm{av}}$ & 0.003 & $\epsilon_{\mathrm{av}}$ & 0.001 & $\epsilon_{\mathrm{av}}$ & 0.007 & $\epsilon_{\mathrm{av}}$ & 0.012 \\
\hline$\hat{c}$ & $\tilde{c}$ & $\hat{c}$ & $\tilde{c}$ & $\hat{c}$ & $\tilde{c}$ & $\hat{c}$ & $\tilde{c}$ & $\hat{c}$ & $\tilde{c}$ \\
\hline 0.448 & 0.453 & 0.408 & 0.412 & 0.356 & 0.331 & 0.383 & 0.368 & 0.326 & 0.305 \\
\hline \multicolumn{2}{|c|}{$\%$ error $=1.1$} & \multicolumn{2}{|c|}{$\%$ error $=0.97$} & \multicolumn{2}{|c|}{$\%$ error $=8.4$} & \multicolumn{2}{|c|}{$\%$ error $=4.1$} & \multicolumn{2}{|c|}{$\%$ error $=6.8$} \\
\hline
\end{tabular}


instantaneous. Cost components are, $c_{1}=1, c_{2}=1.5$, and $c_{3}=6.5$. The transition probabilities are: $p_{0}=p_{1}=p_{2}=$ $0.99, p_{3}=0.1$, and $p_{4}=0.5$. For this example the total number of possible $(K, N)$ pairs is $4 \times 6=24$. For illustration purposes a sample of 5 of them are implemented numerically and shown in Table 2 . We again observe that the limiting probabilities are approximated quite well, with the largest $\epsilon_{\max }$ being 0.031 among the five limiting distributions and the largest percent cost deviation from the simulation results is 8.45 .

Although there is not a straightforward relation between the values of $K^{*}$ and $N^{*}$ and the system parameters, it is observed from the preliminary implementations that the optimal values of $K$ and $N$ are sensitive to the transition probabilities and the exponential rates. To illustrate this sensitivity, let us consider Example 2 again for which the optimal values turn out to be $K^{*}=2$ and $N^{*}=6$. When $v_{3}$ is changed from 1 to 0.1 , the distribution of the sojourn time in state 4 from 3-Erlang to exponential, and $p_{3}$ from 0.1 to 0.99 , the optimal values are changed to be $K^{*}=3$ and $N^{*}=$ 4. This is intuitively expected, since these changes force the components fail at older states. If the changes were such that the probability of failure in the earlier states is increased, we would expect $K^{*}$ to decrease. Indeed, by letting $p_{2}=0.5$ we observed, $K^{*}=2$ and $N^{*}=6$. It is observed that $K^{*}$ tends to take larger values as the failure probabilities in earlier stages get smaller. In another example we considered a model with $M=10$ and $S=8$ where the sojourn times in states $0,1,2$ and 3 are exponential with rates $v_{0}=v_{1}=v_{2}=v_{3}=$ 0.001 , and in states 4, 5 and 6 are distributed as 3-Erlang with rates $v_{4}=v_{5}=v_{6}=3$. Cost parameters were $c_{1}=1$, $c_{2}=1.5, c_{3}=9.5$ and the transition probabilities among states are taken as $p_{0}=p_{1}=p_{2}=p_{3}=p_{4}=0.99, p_{5}=$ $0.7, p_{6}=0.5$. We observed that $K^{*}=3$ and $N^{*}=9$ indicating a smaller number states defining the doubtful set, resulting from small failure probabilities in the first stages.

It is also of interest to see the impact of the number of components in the system. This is investigated by fixing the system and cost parameters and letting $M$ change between 3 and 30 in Example 2. The results are displayed in Table 3. As expected $c^{*}$ and $N^{*}$ increase with $M$ and $K^{*}$ decreases with $M$, which is natural if the system parameters are kept fixed as the number of components increase. In this example system replacement cost was fixed as $M$ increases, which implies that system replacement becomes cheaper relative to the replacement cost of all components individually. This naturally leads to a decrease in the ratio of $N^{*} / M$ for large

Table 3

Example 2, sensitivity of $K^{*}, N^{*}$, and $c^{*}$ w.r.t. $M$

\begin{tabular}{llllc}
\hline$M$ & 3 & 7 & 15 & 30 \\
\hline$K^{*}$ & 3 & 2 & 2 & 1 \\
$N^{*}$ & 2 & 6 & 6 & 17 \\
$C^{*}$ & 0.123 & 0.286 & 0.546 & 0.773 \\
\hline
\end{tabular}

Table 4

Example 2, sensitivity of $K^{*}, N^{*}$, and $c^{*}$ w.r.t. $M, c_{3}=\alpha M c_{1}$

\begin{tabular}{lllllc}
\hline$\alpha$ & $M$ & 3 & 7 & 15 & 30 \\
\hline 0.3 & $K^{*}$ & 1 & 1 & 1 & 1 \\
& $N^{*}$ & 1 & 1 & 9 & 19 \\
& $C^{*}$ & $0.052(64.1)$ & $0.164(51.6)$ & $0.437(39.8)$ & $0.949(34.6)$ \\
0.6 & $K^{*}$ & 1 & 1 & 2 & 2 \\
& $\mathrm{~N}^{*}$ & 1 & 4 & 6 & 12 \\
& $\mathrm{C}^{*}$ & $0.094(35.2)$ & $0.260(23.3)$ & $0.594(18.2)$ & $1.213(16.5)$ \\
0.9 & $\mathrm{~K}^{*}$ & 1 & 2 & 2 & 2 \\
& $\mathrm{~N}^{*}$ & 1 & 4 & 8 & 16 \\
& $\mathrm{C}^{*}$ & $0.078(20.0)$ & $0.217(15.3)$ & $0.485(15.7)$ & $0.984(15.9)$ \\
$\bar{c}$ & & 0.145 & 0.339 & 0.726 & 1.452 \\
\hline
\end{tabular}

values of $M$ indicating that the system is replaced more frequently. In reality, we expect that the system replacement cost increases with the number of components. We implemented this situation by letting $c_{3}=\alpha M c_{1}$ vary as $\alpha$ assumes the values $\alpha=0.3,0.6,0.9$. Results are displayed in Table 4. From this table we observe that larger values of $\alpha$ leads to less frequent system replacements by increasing the value of $N^{*}$ for fixed values of $M$ and $K$.

Since, we aim to reduce the system maintenance costs, an essential question is how much is saved by adopting a group maintenance policy. In Table 4, the figures in parenthesis indicate the percentage savings achieved by using the control policy compared to the cost if components were maintained individually without any system replacement. These figures indicate that the control policy causes significant reductions in the maintenance cost of the system reaching about $64 \%$ for the example undertaken. Also note that the savings are inversely proportional with $M$ and $\alpha$ since as these quantities increase the system replacement is done less frequently and result in less savings due to the control policy.

Finally, we were interested to see the effect of the structure of the transition probabilities among the stages. In maintenance and survival studies usually an increasing failure (hazard) rate is considered for the items under study. However sometimes after the burn-in period, it may also be

Table 5

Example 2, sensitivity of $K^{*}, N^{*}$, and $c^{*}$ w.r.t. $M, c_{3}=0.9 M c_{1}$

\begin{tabular}{clllcc}
\hline$p_{1}$ & $M$ & 3 & 7 & 15 & 30 \\
\hline$\uparrow \downarrow$ & $K^{*}$ & 1 & 1 & 1 & 1 \\
& $N^{*}$ & 2 & 6 & 14 & 29 \\
& $c^{*}$ & 0.168 & 0.375 & 0.774 & 1.544 \\
$\uparrow$ & $K^{*}$ & 2 & 2 & 1 & 1 \\
& $N^{*}$ & 2 & 6 & 14 & 29 \\
& $c^{*}$ & 0.146 & 0.352 & 0.738 & 1.466 \\
& $K^{*}$ & 2 & 2 & 1 & 1 \\
& $N^{*}$ & 2 & 6 & 14 & 29 \\
& $c^{*}$ & 0.150 & 0.348 & 0.705 & 1.402 \\
\hline
\end{tabular}


reasonable to consider decreasing failure rates. Therefore we rerun Example 2 by considering three cases which reflect arbitrary (AFR), increasing (IFR) and decreasing (DFR) failure rates, by changing the transition probabilities correspondingly. For this implementation $c_{3}=0.9 M c_{1}$ is taken and the notation, $(\uparrow \downarrow)$ for AFR; $(\uparrow)$ for IFR, and $(\downarrow)$ for DFR is used. To be more precise, for $(\uparrow \downarrow), p_{0}=$ $0.9, p_{1}=0.5, p_{2}=0.8, p_{3}=0.1, p_{4}=0.6$, for $(\uparrow)$, $p_{0}=0.9, p_{1}=0.8, p_{2}=0.7, p_{3}=0.6, p_{4}=0.4$, for $(\downarrow)$, $p_{0}=0.75, p_{1}=0.80, p_{2}=0.85, p_{3}=0.90, p_{4}=0.95$ are used. The results are displayed in Table 5. From these results it is observed that for all values of $M$ smallest cost is incurred for system where the components are IFR which is followed by DFR systems.

\section{Concluding remarks}

In this study, a maintenance and replacement policy is proposed for a multicomponent, multi-state system, in which both the system and the components can be described through a range of performance levels varying from perfect functioning to complete failure.

The proposed policy suggests a system replacement when the number of the components in doubtful states $K, \ldots, S-2$ is at least $N$ at the time of a preventive or a corrective maintenance of a component, where both $K$ and $N$ are aimed to be optimized. An approximate long run average cost function is proposed under the control policy, which is optimized by numerical methods. The quality of this approximation is assessed by comparing the results with the simulated samples, which implied a satisfactory performance. Several examples are presented to gain an insight about the performance of the control policy. These examples indicated that the control policy achieves significant savings reaching over $\% 60$, over a policy where the components are maintained individually without any system replacement, especially for moderately large systems and when the system replacement cost is low. As the number of components increases while all the other system parameters are kept fixed, the control policy is observed to converge to a simple policy with $K^{*}=1$.

In our model, in order to avoid further complications, a system replacement cost is considered which is independent of the ages of the unfailed machines. A more detailed cost function, which incorporates the component ages, may result in further reductions in the cost figures. The policy proposed in this paper is not necessarily the optimal one and the investigation of the optimal policy for such systems is an open question. A further extension of our model can be done by considering arbitrary sojourn times in the stages.

\section{Appendix A}

Proof of Lemma 1. Let us recall that $E[\cdot \cdot \cdot]$ stands for the conditional expectation, $n_{l}$ for the number of components at state $l, 0 \leq l \leq S-2$ and $n_{\mathrm{D}}$ for the number of components at doubtful states. Below, only the proofs of the parts (b), (e) and (f) are given, and the complete proof can be found in Ref. [14].

$$
\lambda_{N}=\sum_{l=0}^{K-1} E\left[n_{l} \mid n_{\mathrm{D}}=N\right] v_{l}\left(1-p_{l}\right)+E\left[n_{K-1} \mid n_{\mathrm{D}}=N\right] v_{K-1}
$$$$
=\sum_{l=0}^{K-2} \sum_{j=0}^{M-N} \frac{\left(\begin{array}{c}
M \\
N, j, M-N-j
\end{array}\right) \pi_{\mathrm{D}}^{N} \pi_{l}^{j}}{\left(\begin{array}{l}
M \\
N
\end{array}\right) \pi_{\mathrm{D}}^{N} \pi_{\mathrm{G}}^{M-N}}
$$$$
\times\left(\pi_{\mathrm{G}}-\pi_{l}\right)^{M-N-j} j v_{l}\left(1-p_{l}\right)
$$

$$
\begin{aligned}
& +\sum_{j=0}^{M-N} \frac{\left(\begin{array}{c}
M \\
N, j, M-N-j
\end{array}\right) \pi_{\mathrm{D}}^{N} \pi_{K-1}^{j}\left(\pi_{\mathrm{G}}-\pi_{K-1}\right)^{M-N-j}}{\left(\begin{array}{c}
M \\
N
\end{array}\right) \pi_{\mathrm{D}}^{N} \pi_{\mathrm{G}}^{M-N}} \\
& \times j v_{K-1}=\sum_{l=0}^{K-2} \sum_{j=0}^{M-N-1} \frac{\left(\begin{array}{c}
M-N-j \\
j
\end{array}\right) \pi_{l}^{j}\left(\pi_{\mathrm{G}}-\pi_{l}\right)^{M-N-j-1}}{\pi_{\mathrm{G}}^{M-N}} \\
& \times(M-N) \pi_{l} v_{l}\left(1-p_{l}\right) \\
& +\sum_{j=0}^{M-N-1} \frac{\left(\begin{array}{c}
M-N-j \\
j
\end{array}\right) \pi_{K-1}^{j}\left(\pi_{\mathrm{G}}-\pi_{K-1}\right)^{M-N-j-1}}{\pi_{\mathrm{G}}^{M-N}} \\
& \times(M-N) \pi_{K-1} v_{K-1}=\frac{(M-N)}{\pi_{\mathrm{G}}}\left[\omega(K-2)+\pi_{K-1} v_{K-1}\right]
\end{aligned}
$$

For $0 \leq i \leq N$

$$
\begin{aligned}
\mu_{i}= & \sum_{l=K}^{S-3} E\left[n_{l} \mid n_{\mathrm{D}}=i\right] v_{l}\left(1-p_{l}\right)+E\left[n_{S-2} \mid n_{\mathrm{D}}=i\right] v_{S-2} \\
= & \sum_{l=K}^{S-3} \sum_{j=0}^{i} \frac{\left(\begin{array}{c}
M \\
M-i, j, i-j
\end{array}\right) \pi_{\mathrm{G}}^{M-i} \pi_{l}^{j}\left(\pi_{\mathrm{D}}-\pi_{l}\right)^{i-j}}{\left(\begin{array}{c}
M \\
M-i
\end{array}\right) \pi_{\mathrm{G}}^{M-i} \pi_{\mathrm{D}}^{i}} \\
& +\sum_{j=0}^{i} \frac{\left(\begin{array}{c}
M \\
M-i, j, i-j
\end{array}\right) \pi_{\mathrm{G}}^{M-i} \pi_{S-2}^{i}}{\left(\begin{array}{c}
M \\
M-i
\end{array}\right) \pi_{\mathrm{G}}^{M-i} \pi_{\mathrm{D}}^{i}}\left(\pi_{\mathrm{D}}-\pi_{S-2}\right)^{i-j} v_{S-2} \\
= & \sum_{l=K}^{S-3} \sum_{j=0}^{i-1} \frac{i \pi_{l}\left(\begin{array}{c}
i-1 \\
j
\end{array}\right) \pi_{l}^{j}\left(\pi_{\mathrm{D}}-\pi_{l}\right)^{i-j-1}}{\pi_{\mathrm{D}}^{j}} v_{l}\left(1-p_{l}\right) \\
& +\sum_{j=0}^{i} \frac{i \pi_{S-2}\left(\begin{array}{c}
i-1 \\
j
\end{array}\right) \pi_{S-2}^{j}\left(\pi_{\mathrm{D}}-\pi_{S-2}\right)^{i-j-1}}{\pi_{\mathrm{D}}^{i}} v_{S-2}
\end{aligned}
$$


$=\frac{i}{\pi_{\mathrm{D}}}\left[\bar{\omega}(S-3)+\pi_{S-2} v_{S-2}\right]$

For $N+1 \leq i \leq M$

$\beta_{i \delta}=\sum_{l=0}^{S-3} E\left[n_{l} \mid n_{\mathrm{D}}=i\right] v_{l}\left(1-p_{l}\right)+E\left[n_{S-2} \mid n_{\mathrm{D}}=i\right] v_{S-2}$

$=\sum_{l=0}^{K-1} \sum_{j=0}^{M-i} \frac{\left(\begin{array}{c}M \\ i, j, M-i-j\end{array}\right) \pi_{\mathrm{D}}^{i} \pi_{l}^{j}\left(\pi_{\mathrm{G}}-\pi_{l}\right)^{M-i-j}}{\left(\begin{array}{c}M \\ i\end{array}\right) \pi_{\mathrm{D}}^{i} \pi_{\mathrm{G}}^{M-i}}$

$\times j v_{l}\left(1-p_{l}\right)$

$+\sum_{l=K}^{S-3} \sum_{j=0}^{i} \frac{\left(\begin{array}{c}M \\ M-i, j, i-j\end{array}\right) \pi_{\mathrm{G}}^{M-i} \pi_{l}^{j}\left(\pi_{\mathrm{D}}-\pi_{l}\right)^{i-j}}{\left(\begin{array}{c}M \\ M-i\end{array}\right) \pi_{\mathrm{G}}^{M-i} \pi_{\mathrm{D}}^{i}}$

$\times v_{l}\left(1-p_{l}\right)$

$+\sum_{j=0}^{i} \frac{\left(\begin{array}{c}M \\ M-i, j, i-j\end{array}\right) \pi_{\mathrm{G}}^{M-i} \pi_{S-2}^{j}\left(\pi_{\mathrm{D}}-\pi_{S-2}\right)^{i-j}}{\left(\begin{array}{c}M \\ M-i\end{array}\right) \pi_{\mathrm{G}}^{M-i} \pi_{\mathrm{D}}^{i}}$

$\times j v_{S-2}$

$=\sum_{l=K}^{K-1} \sum_{j=0}^{M-i-1} \frac{(M-i) \pi_{l}\left(\begin{array}{c}M-i-1 \\ j\end{array}\right) \pi_{l}^{j}\left(\pi_{\mathrm{G}}-\pi_{l}\right)^{M-i-j-1}}{\pi_{\mathrm{G}}^{M-i}}$

$v_{l}\left(1-p_{l}\right)+\sum_{l=K}^{S-3} \sum_{j=0}^{i-1} \frac{i \pi_{l}\left(\begin{array}{c}i-1 \\ j\end{array}\right) \pi_{l}^{j}\left(\pi_{\mathrm{D}}-\pi_{l}\right)^{i-j-1}}{\pi_{\mathrm{D}}^{i}}$

$\times v_{l}\left(1-p_{l}\right)+\sum_{j=0}^{i-1} \frac{i \pi_{S-2}\left(\begin{array}{c}i-1 \\ j\end{array}\right) \pi_{S-2}^{j}\left(\pi_{\mathrm{D}}-\pi_{S-2}\right)^{i-j-1}}{\pi_{\mathrm{D}}^{i}}$

$\times v_{S-2}=\frac{(M-i)}{\pi_{\mathrm{G}}} \omega(K-1)+\frac{i}{\pi_{\mathrm{D}}}\left[\bar{\omega}(S-3)+\pi_{S-2} v_{S-2}\right]$

\section{References}

[1] Aalen OO. Phase type distribution in survival analysis. Scand J Statist 1995;22:447-63.

[2] Assaf D, Shanthikumar JG. Optimal group maintenance policies with continuous and periodic inspections. Mgmt Sci 1987;33(11):144052.

[3] Balcioğlu B, Gürler Ü. On the use of phase-type distributions for the supplier uncertainty of an inventory system. Submitted for Publication, 2001.

[4] Berk E, Moinzadeh K. Analysis of maintenance policies for $\mathrm{m}$ machines with deteriorating performance. Department of Management Science Working Paper Series, \#5-94, University of Washington, Seattle, 1996.

[5] Gross D, Miller DR. The randomization technique as a modeling tool and solution procedure for transient markov processes. Oper Res 1984;32(2):343-61.

[6] Hsu L. Optimal preventive maintenance policies in a serial production system. Int J Prod Res 1991;29(12):2543-55.

[7] Jansen J, Van Der Duyn Schouten FA. Maintenance optimization on parallel production units. IMA J Math Appl Business Indust 1995;6:113-34

[8] Karlin S, Taylor HM, Fist A. Course in stochastic processes. 2nd ed. New York: Academic Press, 1975.

[9] Kawai H. An optimal maintenance policy of a two-unit standby system. Trans IECE Jpn, E64 1981;9:579-82.

[10] Kumar UD, Knezevic J, Krocker J. Maintenance free operating period - an alternative measure to MTBF and failure rate for specifying reliability. Reliab Engng Syst Saf 1999;64:127-31.

[11] Levitin G, Lisnianski A. Joint redundancy and maintenance optimization for multistate series-parallel systems. Reliab Engng Syst Saf 1999;64:33-42.

[12] Levitin G, Lisnianski A. Optimal separation of elements in vulnerable multi-state systems. Reliab Engng Syst Saf 2001;73:55-66.

[13] van Noortwijk JM, Klatter HE. Optimal inspection decisions for the block mats of the Eastern-Scheldt barrier. Reliab Engng Syst Saf 1999;65:203-11.

[14] Kaya A. A policy for the maintenance of a multistate multicomponent series system. Master's thesis, Bilkent University, 1997.

[15] Özekici S. Optimal periodic replacement of multicomponent reliability systems. Oper Res 1988;36(4):542-52.

[16] Pourret O, Collet J, Bon J. Evaluation of the unavailability of a multistate-component system using a binary model. Reliab Engng Syst Saf 1999;64:13-17.

[17] Ritchken P, Wilson JG. $(m, T)$ group maintenance policies. Mgmt Sci 1990;36(5):632-9.

[18] Van Der Duyn Schouten FA, Ronner T. Calculation of the availability of a two-unit parallel system with cold standby: an illustration of the embedding technique. Prob Engng Info Sci 1989;3:341-53.

[19] Van Der Duyn Schouten FA, Vanneste SG. Analysis and computation of $(n, N)$-strategies for maintenance of a two-component system. Eur $\mathbf{J}$ Oper Res 1990;48:260-74.

[20] Van Der Duyn Schouten FA, Vanneste SG. Two simple control policies for a multicomponent maintenance system. Oper Res 1993;41(6):1125-36.

[21] Van Der Duyn Schouten FA, Wartenhorst P. Transient analysis of a two-unit standby system with markovian degrading units. Mgmt Sci 1994;40:418-28.

[22] Van Der Heijden MC. Interval availability distribution for a 1-out-of2 reliability system with repair. Prob Engng Info Sci 1987;1:211-24. 